\title{
BEATMEN, INTUBIEREN, EXTUBIEREN
}

\section{Künstliche Beatmung zu Hause}

Wenn ein künstlich beatmeter Patient aus der Klinik nach Hause geht, ist das für Ärzte und Pfleger eine Herausforderung. „Bis vor wenigen Jahren waren diese Patienten nur im Krankenhaus. Mittlerweile gehen sie direkt von der Intensivstation in die Häuslichkeit", sagt der Pneumologe Dr. Eckehard Frisch vom Berliner Centrum für Gesundheit (CfG), ein Ärztezentrum der AOK Nordost. Doch die ambulante Versorgung stößt bei diesen Patienten oft an ihre Grenzen.

"Ärztliche Versorgung von außerklinisch beatmeten Patienten findet im Wesentlichen durch Hausärzte, punktuell durch Fachärzte statt, welche kaum Erfahrung im Bereich der außerklinischen Beatmung haben. Damit sind die Patienten gemessen an der Schwere und Komplexität ihrer Erkrankung unterversorgt", so Frisch. Das führt laut AOK Nordost dazu, dass Betroffene wieder stationär eingeliefert werden müssen. Nach Frischs Angaben gibt es auch viele, die länger beatmet werden als nötig. Die Krankenkasse hat daher mit Frisch einen Modellversuch mit dem Titel „Praxis für außerklinische Beatmung" gestartet. Dieses Projekt hat die Deutsche Interdisziplinäre Gesellschaft für Außerklinische Beatmung (DIGAB) nun mit dem 1. Preis für das "Beste Abstract" ausgezeichnet.

Das auf zwei Jahre angelegte Projekt soll die Therapie der be-atmeten Patienten durch Hausbesuche von Spezialisten verbessern. Mindestens einmal pro Quartal kommen ein Pneumologe und eine Atmungstherapeutin zu den Patienten. Zudem sind sie an Werktagen für Patienten, Angehörige und andere Beteiligte erreichbar. Dabei legt Frisch Wert auf die Zusammenarbeit mit Hausarzt, Logopäden, Physiotherapeuten, Pflege und Hilfsmittellieferanten. "Wir sind kein Ersatz für den Hausarzt, sondern eine Ergänzung. Wir sehen uns als Unterstützung und versuchen die Versorgung als Teamspiel aufzustellen", so Frisch. Oft geht es auch um die Versorgung am Lebensende. Da ist es von Vorteil, dass Frisch auch Palliativmediziner ist.

Ärzte Zeitung, 3.9.2015

\section{Optimale Größe für Beatmungstubus gesucht}

Was ist die optimale Größe für den Endotrachealtubus (ETT) bei einer Allgemeinanästhesie? Während man historisch auf möglichst große Durchmesser setzte, gibt es inzwischen einen Trend zu kleineren Durchmessern. Dahinter steckt die Annahme, dadurch Larynxverletzungen, Stimmschäden und Heiserkeit besser vermeiden zu können - gut belegt ist das allerdings nicht. Auch eine jetzt veröffentlichte randomisierte Studie bleibt den Beweis schuldig. Die Verwendung von kleinkalibrigen ETT war bei Schilddrüsen-Op-Patienten weder laryngoskopisch noch klinisch von Vorteil. Schilddrüsen-Operationen sind als Studienobjekt besonders geeignet, weil sie durch die unvermeidbar auftretende Bewegung von Luftröhre und Kehlkopf gegenüber dem fixierten Tubus mit einem besonders hohen Risiko für Schäden an der Larynxmukosa und den Stimmbändern belastet sind.

Insgesamt 49 Patienten mit vollständiger Entfernung oder Lappenresektion der Schilddrüse waren an der Studie beteiligt. Die 24 Patienten der Gruppe 1 wurden dazu mit großen ETT
(Standardgröße 8,0 mm für Männer und 7,5 mm für Frauen), die 25 Patienten der Gruppe 2 mit kleinen ETT (Standardgröße 7,0 mm für Männer und 6,5 mm für Frauen) intubiert.

Die Beurteilung der Stimme mit dem GRBAS-Score (Grad, Rauheit, Behauchtheit, Asthenie, Pressen) 24 Stunden und drei Wochen nach der Op förderte in keinem Teilaspekt signifikante Veränderungen gegenüber dem präoperativen Status zutage. Laryngoskopische Befunde waren jedoch an Tag 1 signifikant häufiger: Erytheme, Ödeme, Polypen/Knoten und/oder eine verminderte Beweglichkeit der Stimmbänder wurden bei 41 Patienten beobachtet. Vor dem Eingriff hatten nur sechs Patienten auffällige Befunde gehabt, vor allem in Form von milden Erythemen. Die meisten der postoperativen Schäden waren innerhalb von drei Wochen abgeklungen, Mukosaveränderungen fanden sich zu diesem Zeitpunkt noch bei 14 Patienten.

Ob die Patienten mit klein- oder großkalibrigen ETT intubiert worden waren, hatte jedoch auf keinen dieser Parameter einen Einfluss. In Bezug auf Schmerzen und die Selbstbeurteilung der Stimme ergaben sich ebenfalls keine Unterschiede zwischen den Gruppen 1 und 2.

Die Studienautoren um Rania Mehanna von der Universitätsklinik in Cork müssen daher eingestehen, dass ihre Hypothese, mit kleinkalibrigen ETT weniger Larynxtraumen zu erzeugen, durch die Ergebnisse nicht gestützt wird. Allerdings vermuten sie, dass die Studie möglicherweise zu klein war, um solche Effekte demonstrieren zu können.

Mehanna R et al. Effect of Endotracheal Tube Size on Vocal Outcomes After Thyroidectomy: A Randomized Clinical Trial. JAMA Otolaryngol Head Neck Surg, online 9. Juli 2015; doi:10.1001/jamaoto.2015.1198

\section{Musik unterstützt Entwöhnung von Beatmung}

Bei der Entwöhnung von einer längeren Beatmungstherapie kann es hilfreich sein, den Patienten Musik seiner Wahl per Kopfhörer hören zu lassen. Dies beruhigt den Patienten in vielerlei Hinsicht: Die Herz- und Atemfrequenz sinken, Dyspnoe und Angst mildern sich und auch die Entwöhnungsphase wird verkürzt.

Forscher der Universität Pittsburgh/ Pennsylvania hatten 28 Patienten nach längerer Beatmung während einer sechstägigen Entwöhnung vom Beatmungsgerät jeden zweiten Tag Musik hören lassen. Auf diese Weise konnten sie bei jedem Patienten die Parameter an Tagen mit oder ohne Musik messen.

Ihr Fazit: Der Einsatz der Musik ist einfach und möglicherweise sinnvoll, um die Entwöhnung zu erleichtern.

Jahrestagung der American Thoracic Society, Denver/USA 2015 